Bài báo khoa học

\title{
Nghiên cứu xây dựng hoàn thiện quy trình xuất bản điện tử và công cụ quản lý Tạp chí Khí tượng Thủy văn
}

\section{Đoàn Quang Trí1, Đinh Việt Hoàng ${ }^{1^{*}}$}

1 Tạp chí Khí tượng Thủy văn, Tổng cục Khí tượng Thủy văn; doanquangtrikttv@gmail.com; hoang.ceid@gmail.com;

* Tác giả liên hệ: hoang.ceid@gmail.com; Tel.: +84-931769823

Ban Biên tập nhận bài: 23/11/2020; Ngày phản biện xong: 02/03/2021; Ngày đăng bài: $25 / 03 / 2021$

Tóm tắt: Quá trình phát triển công nghệ số và hội nhập quốc tế đặt ra những yêu cầu cần hoàn thiện công cụ khai thác quản lý và quy trình xuất bản điện tử Tạp chí Khí tượng Thủy văn (KTTV). Quy trình xuất bản điện tử được mô hình hóa bằng ngôn ngữ lập trình $\mathrm{PHP}$ và lưu trữ dữ liệu người dùng, bản thảo bài báo, quá trình phản biện của người dùng thông qua hệ quản trị cơ sở dữ liệu MySQL. Các công cụ quản lý với những thao tác và giao diện hiển thị sử dụng HTML kết hợp với CSS và Javascript. Kết quả nghiên cứu đã xây dựng thành công quy trình xuất bản điện tử cùng với các công cụ quản lý hỗ trợ cho tác giả, Ban biên tập nhà phản biện, Tổng biên tập và biên tập viên trong công tác xuất bản điện tử Tạp chí KTTV. Các công cụ quản lý và quy trình xuất bản điện tử cho thấy sự phù hợp, hiệu quả trong công tác xuất bản điện tử Tạp chí KTTV.

Từ khóa: Công cụ quản lý; Quy trình xuất bản điện tử; Tạp chí Khí tượng Thủy văn.

\section{Mở đầu}

Thế kỷ 21 nổi lên như thời đại của khoa học, công nghệ và những đột phá mạnh mẽ trong hành trình đào sâu hơn về những bí ẩn thế giới của con người. Chính vì vậy, các tin tức được cập nhật thường xuyên sẽ giúp cho chúng ta không bỏ lỡ những thành tựu tuyệt vời nhất và trau dồi kiến thức của mình. Các trang thông tin điện tử của các tạp chí khoa học cũng ngày càng phát triển và đáp ứng được nhu cầu tìm kiếm thông tin cũng như gửi bài, đăng tải bài báo khoa học trực tuyến của tác giả trên toàn thế giới. Những thông tin khoa học cung cấp cho các độc giả, phải kể đến sự đóng góp của những hệ thống nhà xuất bản quốc tế uy tín như: Springer [1], Elsevier [2], Wiley-Blackwell [3], Taylor\&Francis [4], Sage [5], Oxford University Press [6], Cambridge University Press [7],... Viện Thông tin khoa học (Institute for Scientific Information, ISI) của Mỹ đã đề xuất sự phân loại các tạp chí khoa học trên thế giới theo chất lượng và được thừa nhận rộng rãi. Chỉ số ISI đang được dùng như một thước đo quan trọng để đánh giá chất lượng nghiên cứu tại một số nước đang phát triển, trong đó có Việt Nam [8]. ISI bao gồm tập hợp các tạp chí trong lĩnh vực khoa học tự nhiên và công nghệ (SCI, SCIE), khoa học xã hội (SSCI) và khoa học nhân văn và nghệ thuật (AHCI). Đối với những nhà xuất bản quốc tế uy tín, những Tạp chí quốc tế uy tín cũng đều xây dựng cho mình những quy trình xuất bản điện tử riêng với những hệ thống quản lý ưu việt cho người sử dụng, độc giả, nhà phản biện, biên tập viên, hội đồng biên tập, tổng biên tập,... Trong nước hiện nay cũng có rất nhiều tạp chí khoa học đã xây dựng quy trình xuất bản điện tử cho các bài báo khoa học với mục tiêu đơn giản hóa quy trình xuất bản, chuyển từ phương thức xuất bản tạp chí ở dạng bản giấy sang bản điện tử nhằm cung cấp các sản phẩm khoa học là các số xuất bản, các bài báo khoa học một cách nhanh chóng và tiện lợi [9-12]. Tạp chí Khí tượng Thủy 
văn (KTTV) là một tạp chí khoa học chuyên ngành uy tín và lâu đời với số đầu tiên xuất bản vào ngày 20/4/1956 do Giám đốc Nha Khí tượng GS. Nguyễn Xiển làm chủ biên. Trải qua 65 năm xuất bản với hơn 723 số xuất bản tới nay, Tạp chí KTTV duy trì xuất bản định kỳ 12 số tiếng Việt hàng tháng. Tháng 12 năm 2018, Tạp chí Khí tượng Thủy văn xuất bản số tiếng Anh đầu tiên, đến nay, tạp chí đã duy trì xuất bản 03 số định kỳ Tiếng Anh và số lượng tăng dần theo từng năm. Tạp chí KTTV với mục tiêu nâng cao chất lượng xuất bản uy tín cả về chất lượng và số lượng đồng thời chuyển dần từ xuất bản dạng in sang xuất bản điện tử toàn bộ các số định kỳ tiếng Việt và tiếng Anh [13-14]. Tạp chí KTTV đã không ngừng nghiên cứu, hoàn thiện, trao đổi và làm việc với các tạp chí uy tín trong và ngoài nước để chuẩn hóa và hoàn thiện các form mẫu, quy trình xuất bản, trang thông tin điện tử của Tạp chí nhằm bắt kịp xu thế hội nhập quốc tế trong thời kỳ cách mạng công nghệ 4.0. Việc không ngừng phát triển và nâng cao chất lượng xuất bản, trong năm 2020 Tạp chí KTTV đã được Hội đồng Giáo sư liên ngành Khoa học Trái đất - Mỏ công nhận nâng điểm từ $0-0,5$ lên $0-0,75$ điểm từ năm 2020 [15]. Tạp chí KTTV đang tiếp tục cố gắng phát triển hoàn thiện hơn nữa và đặt ra mục tiêu được Hội đồng Giáo sư liên ngành Khoa học Trái đất - Mỏ công nhận 1,0 điểm trong năm 2022. Bên cạnh đó, Hội đồng Giáo sư ngành Thủy Lợi vẫn đánh giá và duy trì điểm của Tạp chí KTTV từ $0-1,0$ điểm. Để đạt được mục tiêu trên, Tạp chí đã xây dựng và hoàn thiện quy trình xuất bản điện tử với các công cụ hỗ trợ cho các tác giả, nhà phản biện, Ban biên tập có thể gửi bài, nhận bài, phản biện online trên hệ thống thay việc gửi bài, nhận bài, mời chuyên gia phản biện, trao đổi, liên hệ... tại Tạp chí KTTV hoàn toàn thông qua hòm thư điện tử. Trong bối cảnh nhu cầu đăng bài trên tạp chí ngày càng tăng, trong khi công tác gửi bài, nhận bài, phản biện ... thông qua hòm thư điện tử đã không còn phù hợp và cần có một giải pháp để giải quyết triệt để những vấn đề: lưu trữ quá trình xuất bản; tìm kiếm, khai thác thông tin; thống kê bài báo; theo dõi quá trình xử lý bài báo; không bị ảnh hưởng bởi chính sách của nhà cung cấp dịch vụ là yêu cầu hết sức cấp thiết. Mục đích của nghiên cứu cần xây dựng một công cụ quản lý và quy trình xuất bản điện tử để nâng cao hiệu quả công tác xuất bản Tạp chí đáp ứng được nhu cầu phát triển trong thời kỳ cách mạng công nghệ 4.0.

\section{Phương pháp nghiên cứu}

\subsection{Phưong pháp xây dựng cơ sở dũ liệu}

Thiết kế cấu trúc dữ liệu là một thành phần rất quan trọng trong quy trình xuất bản điện tử, việc thiết kế cơ sở dữ liệu mạch lạc, toàn vẹn sẽ giúp tối ưu được việc xử lý quy trình trên các bàn làm việc của tác giả, nhà phản biện và Ban Biên tập. Với yêu cầu liên kết dữ liệu chặt chẽ, bảo đảm tính thống nhất, toàn vẹn thì hệ cơ sở dữ liệu quan hệ có cấu trúc theo chuẩn SQL là lựa chọn tốt nhất. Vì vậy, trong nghiên cứu này đã lựa chọn hệ quản trị cơ sở dữ liệu MySQL [16-17] là hệ cơ sở dữ liệu quan hệ tương thích với ngôn ngữ lập trình PHP cả về tốc độ lẫn việc triển khai xây dựng ứng dụng Web. 


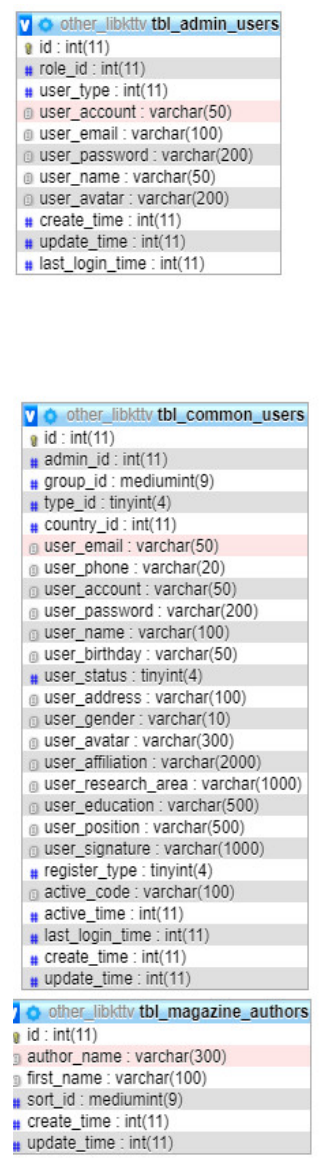

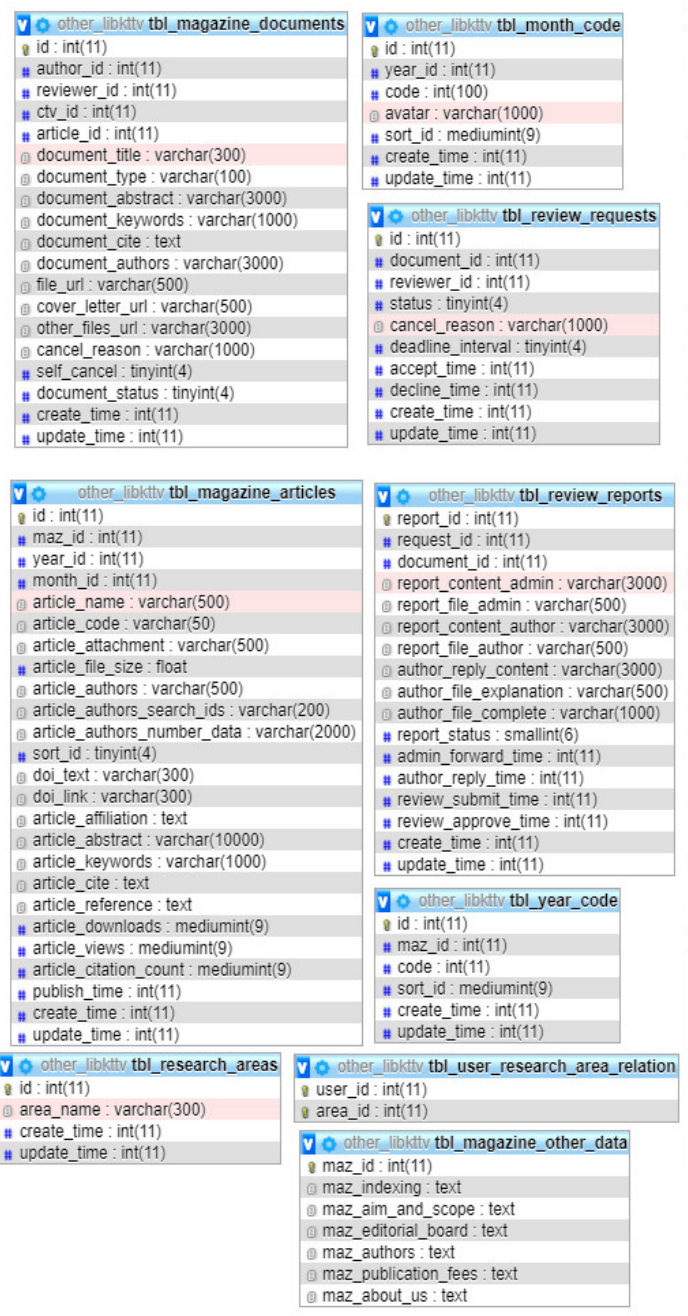

Hình 1. Cấu trúc bảng cơ sở dữ liệu.

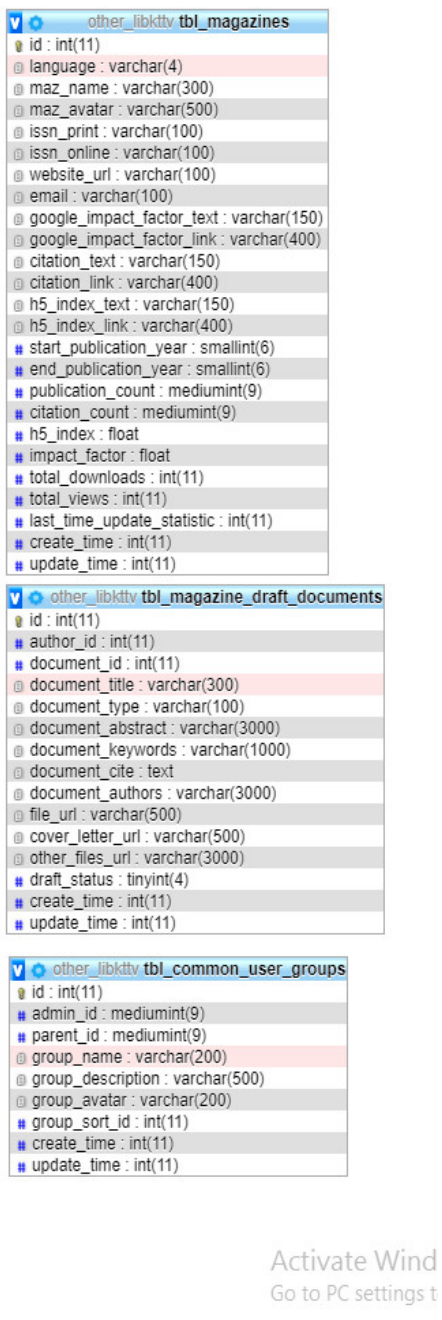

- Nhóm bảng dữ liệu liên quan đến việc quản trị website và Ban biên tập như:

tbl_admin_users, tbl_magazines_articles, tbl_magazines, tbl_year_codes, tbl_month_codes, tbl magazine authors...;

- Nhóm bảng dữ liệu liên quan đến tài khoản tác giả, nhà phản biện: tbl_common_users, tbl_common_user_groups, tbl_research_area, tbl_research_area_relation;

- Nhóm bảng dữ liệu liên quan đến quy trình sơ loại: tb̉ l_magazine_draft_documents, tbl magazine documents;

- Nhóm bảng dữ liệu liên quan đến quy trình phản biện: tbl_review_reports, tbl_review_requets.

\subsection{Phương pháp xây dụng công cu quản lý và quy trình xuất bản điện tử}

Quy trình xuất bản điện tử gồm 4 phần chính: Phần thứ nhất là Đăng ký, gửi bài dành cho tác giả đăng ký tài khoản và gửi bài đến tòa soạn; Phần thứ 2 là Sơ loại dành cho hội đồng biên tập sơ loại bài báo; Phần thứ 3 là Bình duyệt dành cho công tác phản biện bài báo và duyệt xuất bản; Phần thứ 4 là Xuất bản dành cho các biên tập viên của tòa soạn tiến hành biên tập chỉnh sửa và xuất bản bài báo. Quy trình xuất bản điện tử được mô hình hóa bằng ngôn ngữ lập trình PHP [18] và lưu trữ dữ liệu người dùng, bản thảo bài báo, quá trình phản biện của người dùng thông qua hệ quản trị cơ sở dữ liệu MySQL. Các công cụ quản lý với những thao tác và giao diện hiển thị sử dụng HTML kết hợp với CSS [19] và Javascript [20]. Đây là những ngôn ngữ cơ bản và cốt lõi của các ứng dụng Web phù hợp với mục tiêu của đề tài đặt 
ra, dễ dàng cho việc sử dụng, bảo trì và đảm bảo tính toàn vẹn, bảo mật của hệ thống. Sơ đồ Quy trình xuất bản điện tử được thực hiện cụ thể như (Hình 2).

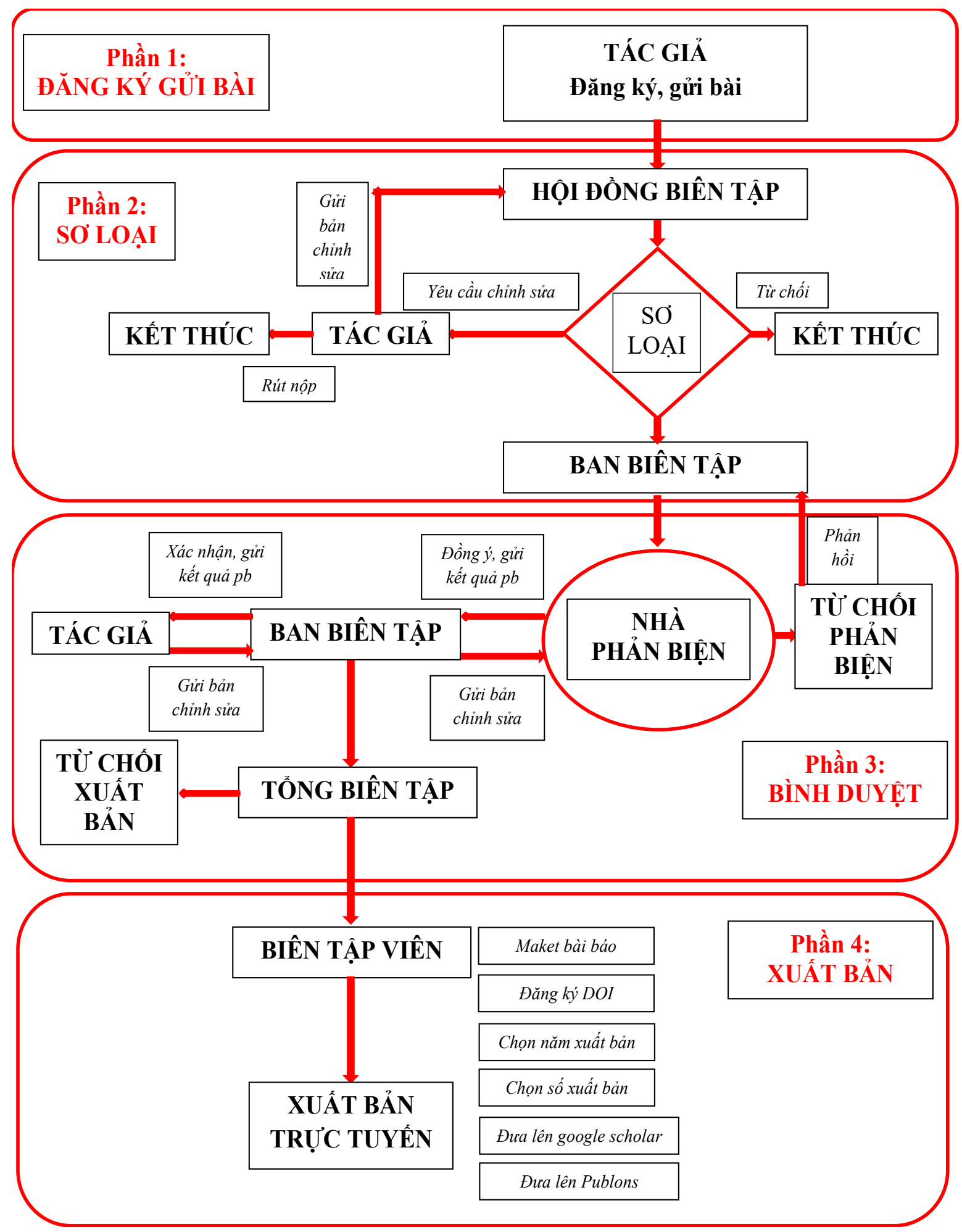

Hình 2. Sơ đồ quy trình xuất bản điện tử Tạp chí KTTV.

Các công cụ quản lý đáp ứng các yêu cầu chính sau: (1) Giao diện hiển thị trực quan, dễ sử dụng; (2) Đảm bảo vận hành đầy đủ theo các bước của quy trình xuất bản điện tử Tạp chí KTTV; (3) Lưu trữ toàn bộ các bài báo, tác giả, nhà phản biện và quá trình phản biện trong cơ sở dữ liệu; (4) Tự động thống kê được số lượng lượt tải, lượt xem, lượt trích dẫn, tỉ lệ từ chối đăng, tỉ lệ rút nộp. 


\section{Kết quả và thảo luận}

\subsection{Công cu quản lý dành cho tác giả}

Quy trình xuất bản điện tử được xây dựng, sau khi đăng ký tài khoản và đăng nhập hệ thống, tác giả sẽ đăng nhập vào bàn làm việc với nhiều chức năng để quản lý tài khoản. Công cụ quản lý dành cho tác giả với giao diện trực quan, dễ hiểu, tiện cho việc gửi bài và theo dõi quá trình xử lý bài báo cho đến khi bài báo được xuất bản online. Tác giả có thể gửi bài báo đến tòa soạn với chức năng Đăng bài mới. Sau khi điền đầy đủ các trường thông tin, đính kèm file bài báo, tác giả có thể gửi bài báo ngay hoặc lưu thông tin và rà soát lại trước khi gửi tòa soạn. Như vậy, tác giả có thể gửi bài mới, theo dõi quá trình sơ loại, quá trình phản biện, quá trình biên tập và xuất bản bài báo đồng thời có thể nhận thông tin phản hồi, chỉnh sửa bài báo và gửi lại cho tòa soạn thông qua bàn làm việc trên (Hình 3 ).

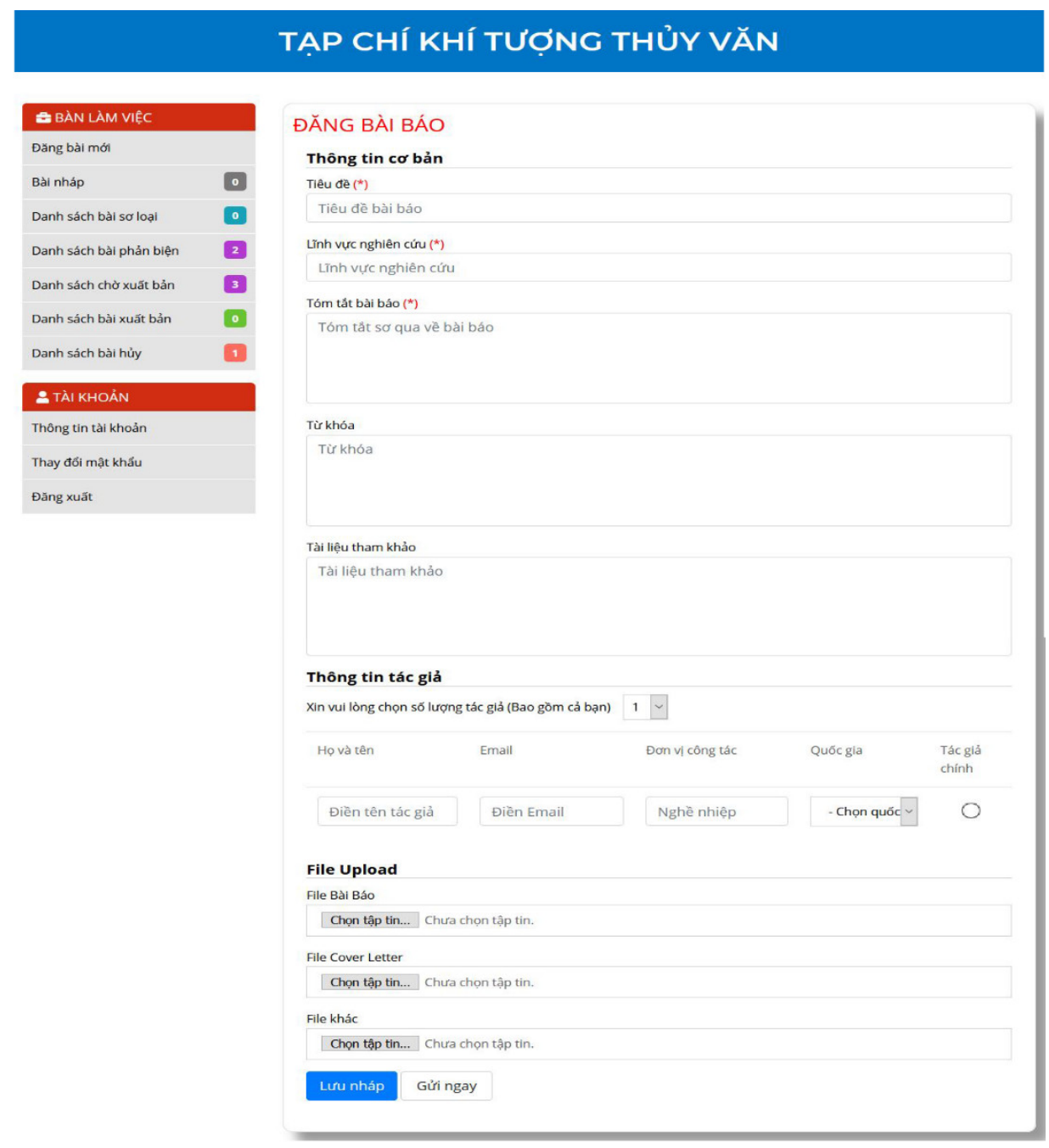

Hình 3. Công cụ quản lý dành cho tác giả.

\subsection{Công cu quản lý dành cho Ban biên tập}

Để nhận được bài báo của tác giả, tiến hành công tác sơ loại, mời phản biện, nhận kết quả phản biện, yêu cầu tác giả chỉnh sửa, phân công biên tập, biên tập chỉnh sửa và xuất bản online, Ban biên tập cần có một giao diện được gọi là Bàn làm việc với nhiều chức năng để thực hiện các công việc đó. Trong quy trình xuất bản điện tử, sau khi tác giả gửi bài, thành viên Hội đồng biên tập sẽ nhận được bài báo và tiến hành sơ loại bài báo (Hình 4). 


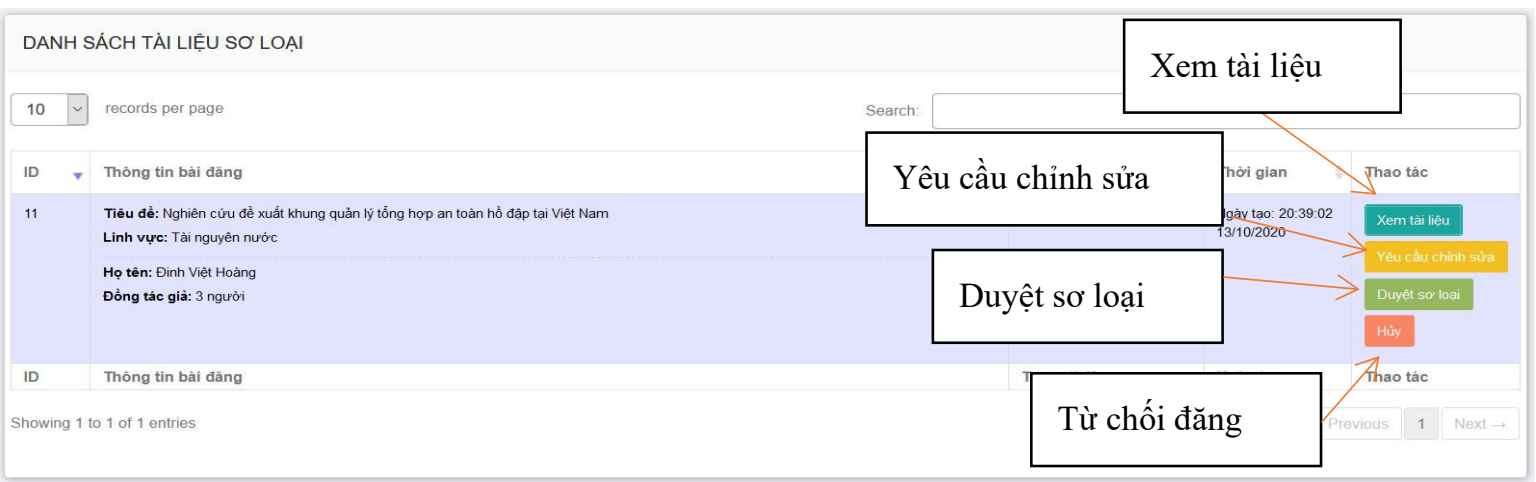

Hình 4. Công tác sơ loại bài báo.

Tại bàn làm việc, Hội đồng biên tập có thể xem toàn bộ các tài liệu của tác giả gửi đến và tiến hành sơ loại bài báo như sau: Trường hợp thứ nhất, bài báo có nhiều lỗi, chưa đúng Form xuất bản, Hội đồng biên tập yêu cầu tác giả chỉnh sửa. Tác giả có thể chỉnh sửa theo yêu cầu và gửi lại bài báo đã chỉnh sửa kèm với bản giải trình hoặc rút nộp bài báo nếu không chỉnh sửa được. Trường hợp thứ hai, đối với các bài báo đã đảm bảo chất lượng, Hội đồng biên tập sẽ duyệt sơ loại để gửi Ban biên tập mời phản biện cho bài báo. Trường hợp thứ ba, các bài báo không đạt tiêu chuẩn, không phù hợp với các lĩnh vực nghiên cứu của Tạp chí, Hội đồng biên tập sau khi xem xét, trình Tổng Biên tập và ra thông báo từ chối đăng bài. Sau khi duyệt sơ loại, bài báo được chuyển cho người phụ trách Ban biên tập để tiến hành mời nhà phản biện được thể hiện trên (Hình 5).

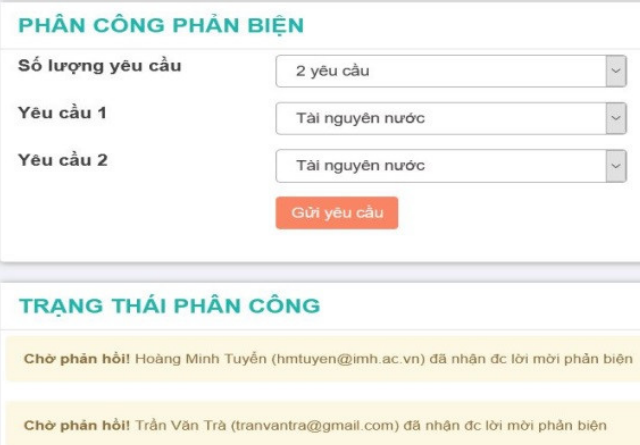

Hình 5. Ban biên tập mời phản biện.

Ban biên tập sẽ mời hai nhà phản biện đối với mỗi bài báo khoa học theo đúng với lĩnh vực chuyên ngành. Sau khi gửi yêu cầu, ban biên tập sẽ đợi phản hồi từ nhà phản biện, nếu 2 nhà phản biện đồng ý, trạng thái phân công sẽ chuyển sang trạng thái đồng ý phản biện. Nếu nhà phản biện từ chối, trạng thái phân công chuyển sang từ chối, lúc này Ban biên tập sẽ mời nhà phản biện khác cho bài báo.

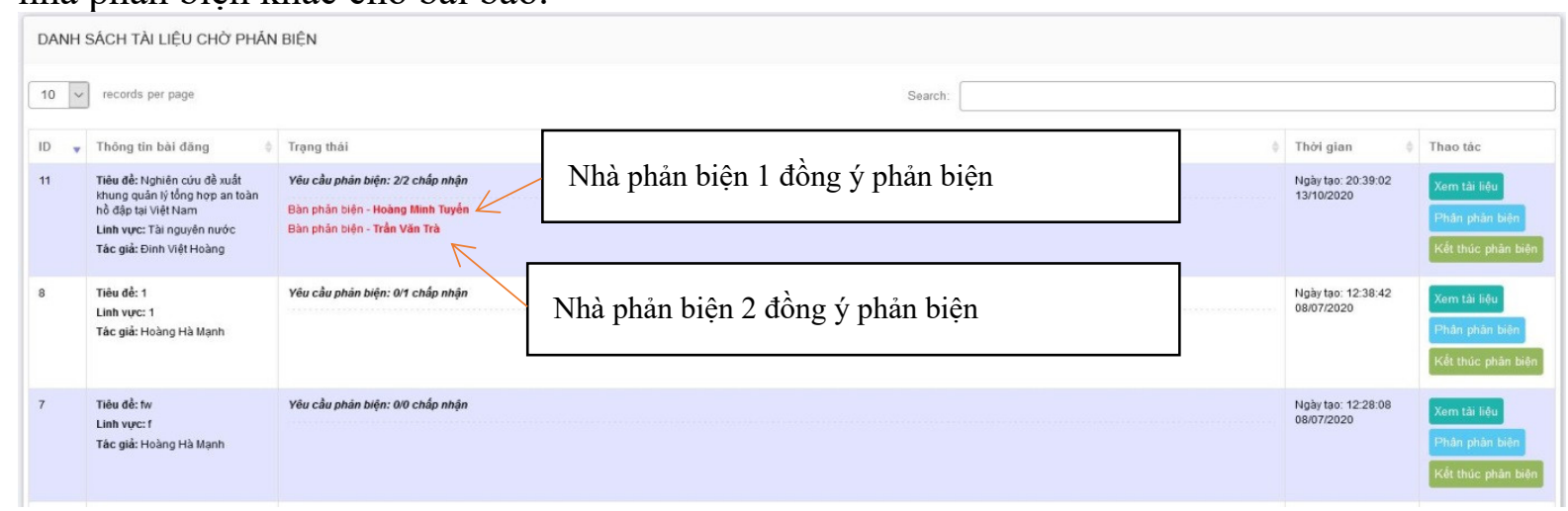

Hình 6. Nhà phản biện đồng ý phản biện bài báo. 
Bàn làm việc của Ban biên tập cập nhật trạng thái đồng ý phản biện và đợi nhà phản biện trả kết quả phản biện.

\begin{tabular}{|c|c|c|c|c|}
\hline \multicolumn{4}{|c|}{ BÁN LÁM VIẸC CÜA PHÁN BIỆN } & \multirow{2}{*}{ Kết quả chỉnh sửa } \\
\hline REPORT SO 1 [ID: 7$]$ & & & & \\
\hline PHAN BIẸN & & \multirow{4}{*}{ 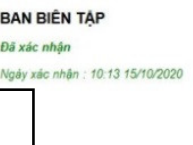 } & \multirow{4}{*}{\multicolumn{2}{|c|}{ 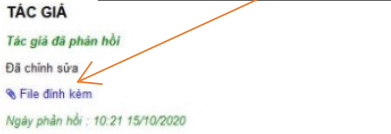 }} \\
\hline 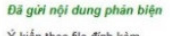 & & & & \\
\hline 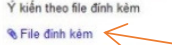 & & & & \\
\hline 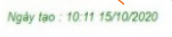 & Yêu cầu chỉnh sửa & & & \\
\hline
\end{tabular}

Hình 7. Nội dung chi tiết quá trình phản biện.

Đối với mỗi nhà phản biện, sau khi gửi kết quả phản biện và file yêu cầu chỉnh sửa, Ban biên tập sẽ xác nhận và gửi thông báo đến tác giả. Tác giả nhận được yêu cầu chỉnh sửa sẽ tiến hành chỉnh sửa bài báo rồi gửi lại kết quả cho Ban biên tập và nhà phản biện. Quá trình phản biện sẽ lặp lại cho đến khi hai nhà phản biện đồng ý với kết quả chỉnh sửa cuối cùng của tác giả và kết thúc quy trình phản biện. Tiếp theo bài báo sẽ được chuyển tới Tổng biên tập để duyệt xuất bản và phân công cho các biên tập viên chỉnh sửa và xuất bản online.

\subsection{Công cư quản lý dành cho nhà phản biện}

Nhà phản biện cần đăng nhập tài khoản trên hệ thống và vào bàn làm việc của mình. Bàn làm việc của nhà phản biện với giao diện đơn giản, đầy đủ các chức năng giúp cho nhà phản biện dễ dàng nhận yêu cầu phản biện từ Ban Biên tập, xem các thông tin cơ bản của bài báo và file đính kèm bài báo hoàn chỉnh, trả kết quả phản biện, duyệt kết quả chỉnh sửa, kết thúc phản biện.

\section{TAP CHÍ KHÍ TƯợNG THỦY VĂN}

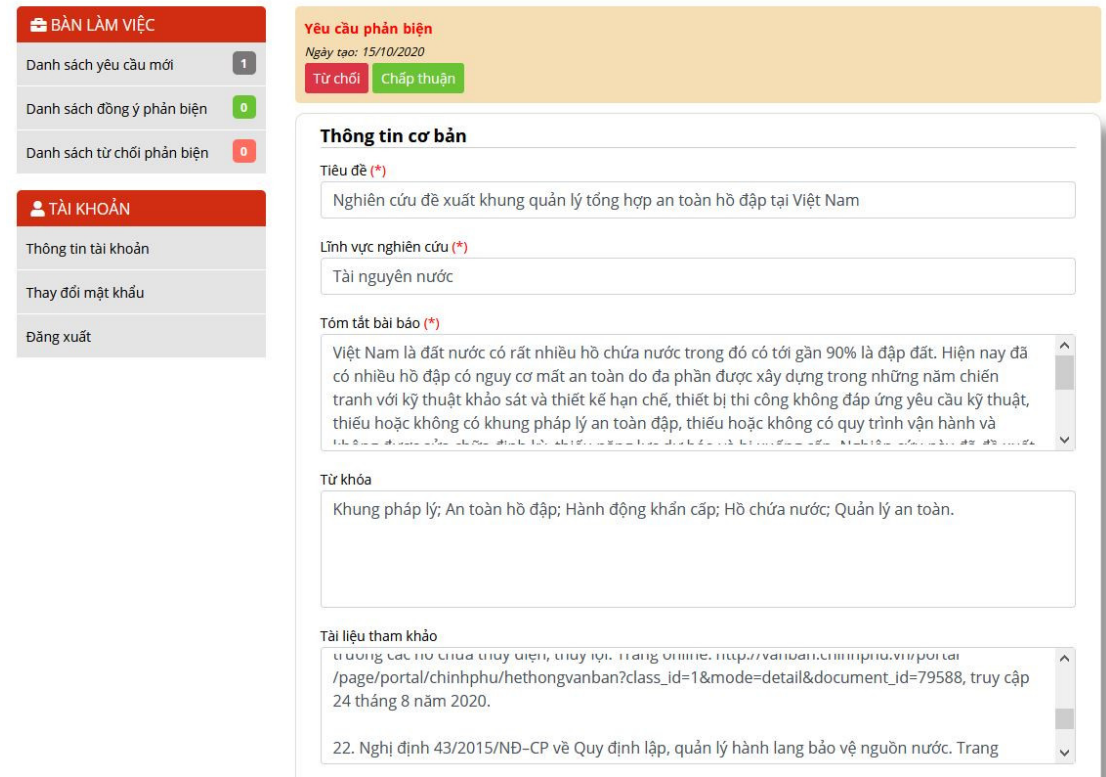

Hình 8. Công cụ quản lý dành cho nhà phản biện.

Nhà phản biện nhận được yêu cầu phản biện từ Ban biên tập, nhà phản biện có thể từ chối hoặc đồng ý phản biện. Nếu từ chối, bài báo sẽ chuyển sang danh sách từ chối phản biện và có thông báo cho Ban biên tập. Nếu chấp thuận, bài báo sẽ chuyển tới danh sách đồng ý phản 
biện và nhà phản biện có 15 ngày để phản biện bài báo (Hình 8$)$. Sau khi phản biện xong, nhà phản biện sẽ gửi lại phiếu nhận xét và yêu cầu chỉnh sửa cho Ban biên tập và tác giả (Hình 9).

\section{TẠP CHÍ KHÍ TƯợNG THỦY VĂN}
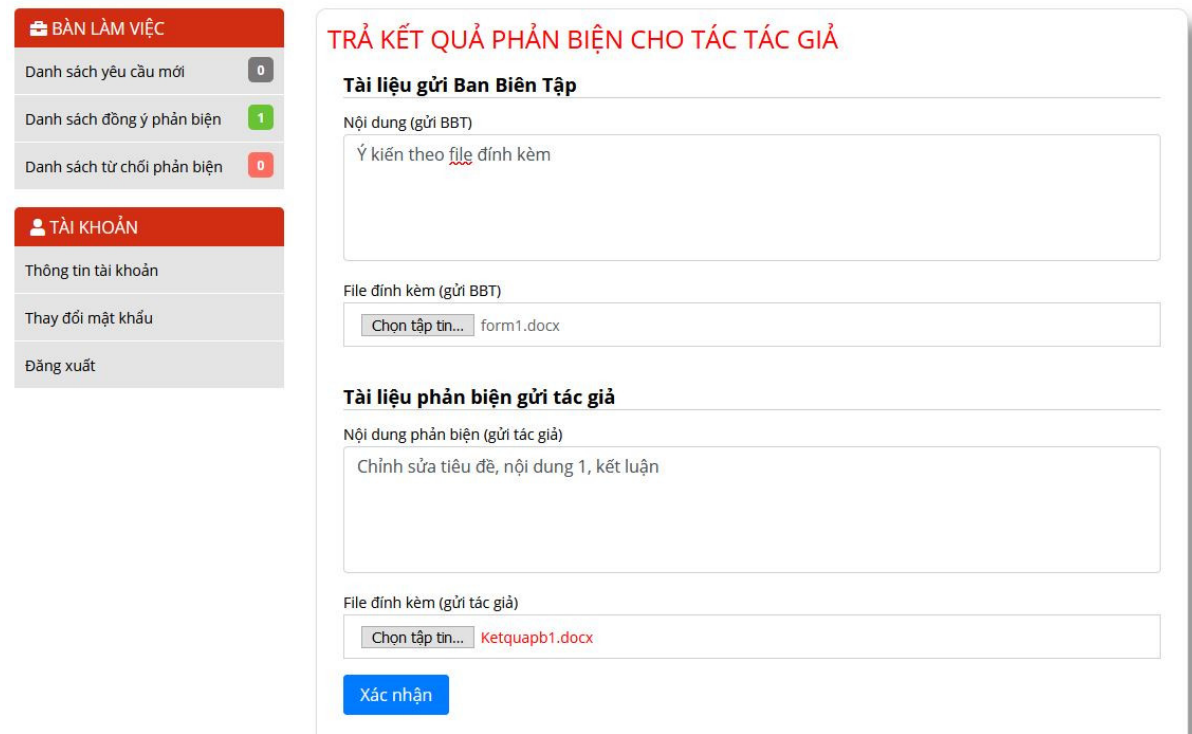

Hình 9. Nhà phản biện gửi kết quả phản biện.

Sau khi nhận được kết quả phản biện, Ban biên tập xác nhận để gửi yêu cầu chỉnh sửa cho tác giả. Tác giả sẽ chỉnh sửa và gửi lại, lúc này nhà phản biện có thể xem bản chỉnh sửa của tác giả (Hình 10).

\section{TẠP CHÍ KHÍ TƯợNG THỦY VĂN}

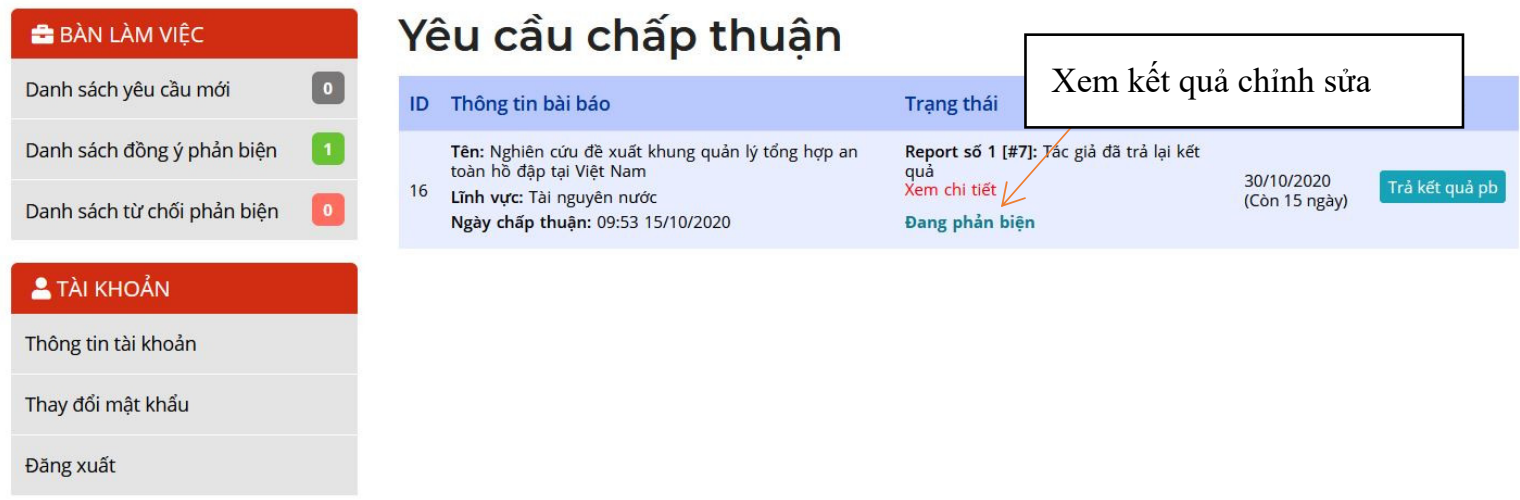

Hình 10. Nhà phản biện nhận bài báo đã chỉnh sửa.

Nếu nhà phản biện chưa đồng ý với kết quả chỉnh sửa có thể tiếp tục gửi yêu cầu chỉnh sửa cho tác giả. Nhà phản biện đồng ý với kết quả chỉnh sửa sẽ kết thúc quá trình phản biện.

\subsection{Các công cu hỗ trọ̣ quản lý luu trũu, tìm kiếm, thống kê}

Tất cả các bài báo, tác giả, nhà phản biện và quá trình phản biện đều được lưu lại và phân tách cụ thể giúp Ban biên tập dễ dàng quản lý, khai thác, tìm kiếm và sử dụng một cách hiệu quả. 


\begin{tabular}{|c|c|c|c|c|c|c|c|c|c|c|}
\hline \multicolumn{4}{|c|}{ Tiêu đề bài báo } & \multicolumn{2}{|l|}{ Tạp chi } & \multicolumn{5}{|l|}{ Số năm } \\
\hline \multicolumn{4}{|c|}{ Timm kiếm theo tên bài báo } & \multicolumn{2}{|c|}{ - Chọn tạp chí -- } & \multicolumn{5}{|c|}{-- Chọn số năm - } \\
\hline \multicolumn{3}{|c|}{ Số tháng } & Sắp xếp theo & \multicolumn{2}{|c|}{ Ngày tạo } & & & & & \\
\hline \multicolumn{6}{|c|}{ DANH SÁCH BÀl BÁO KHOA HỌC (1733 BÀl) } & & & \multicolumn{3}{|c|}{ - Thao tác khác- } \\
\hline$\square$ & ID & Thông tin bäi bảo & & & Thông tin tạp chí & \multicolumn{2}{|c|}{ Ngày tạo } & \multicolumn{3}{|c|}{ Thao tác } \\
\hline$\square$ & 1777 & 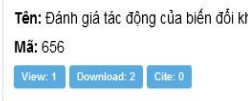 & ậu lên dòng chày lưu vục sông Đồng Nai & & $\begin{array}{l}\text { Tạp chi: Tạp chi Khi tượng Thùy văn } \\
\text { Số năm: } 2015 \\
\text { Số tháng: } 8\end{array}$ & $\begin{array}{l}\text { Ngày } \\
20 / 01 \\
\text { Xuất } \\
01 / 08\end{array}$ & $\begin{array}{l}\text { tạo : } 10: 55 \\
2021 \\
\text { oản : } 06: 25 \\
2015\end{array}$ & 0 & $\mathbb{8}$ & 魚 \\
\hline$\square$ & 1775 & 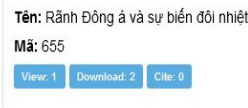 & Strong thời hỳ mùa đông ở Việt Nam & & $\begin{array}{l}\text { Tạp chi: Tạp chí Khi tượng Thủy văn } \\
\text { Số năm: } 2015 \\
\text { Số thaing: } 7\end{array}$ & $\begin{array}{l}\text { Ngày } \\
20 / 01 \\
\text { Xuất } \\
12 / 07\end{array}$ & $\begin{array}{l}\text { tạo : } 10: 33 \\
2021 \\
\text { oản : } 09: 05 \\
2015\end{array}$ & o & $\mathbb{Z}$ & 田 \\
\hline$\square$ & 1774 & 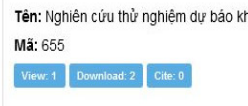 & lậu từ mỏ hinh CFSV2 bằng phương pháp DOWNSCALING Thống & & $\begin{array}{l}\text { Tạp chi: Tạp chi Khi tượng Thủy văn } \\
\text { Số năm: } 2015 \\
\text { Số tháng: } 7\end{array}$ & $\begin{array}{l}\text { Ngày } \\
20 / 01 \\
\text { xuất } \\
\text { 06/07 }\end{array}$ & $\begin{array}{l}\tan : 10: 22 \\
2021 \\
\text { oàn : } 06 \cdot 10 \\
2015\end{array}$ & 0 & $\square$ & 田 \\
\hline
\end{tabular}

Hình 11. Giao diện danh sách các bài báo đã xuất bản.

Tạp chí KTTV đã tiến hành cập nhật các bài báo từ năm 2000 đến nay với khoảng 1.733 bài báo và lưu trữ trong danh sách các bài báo đã xuất bản. Tại đây, các Biên tập viên và thành viên Ban biên tập có thể tìm kiếm các bài báo một cách nhanh chóng theo tiêu đề, theo số năm xuất bản, số tháng xuất bản,... với kết quả được thể hiện trên (Hình 11).

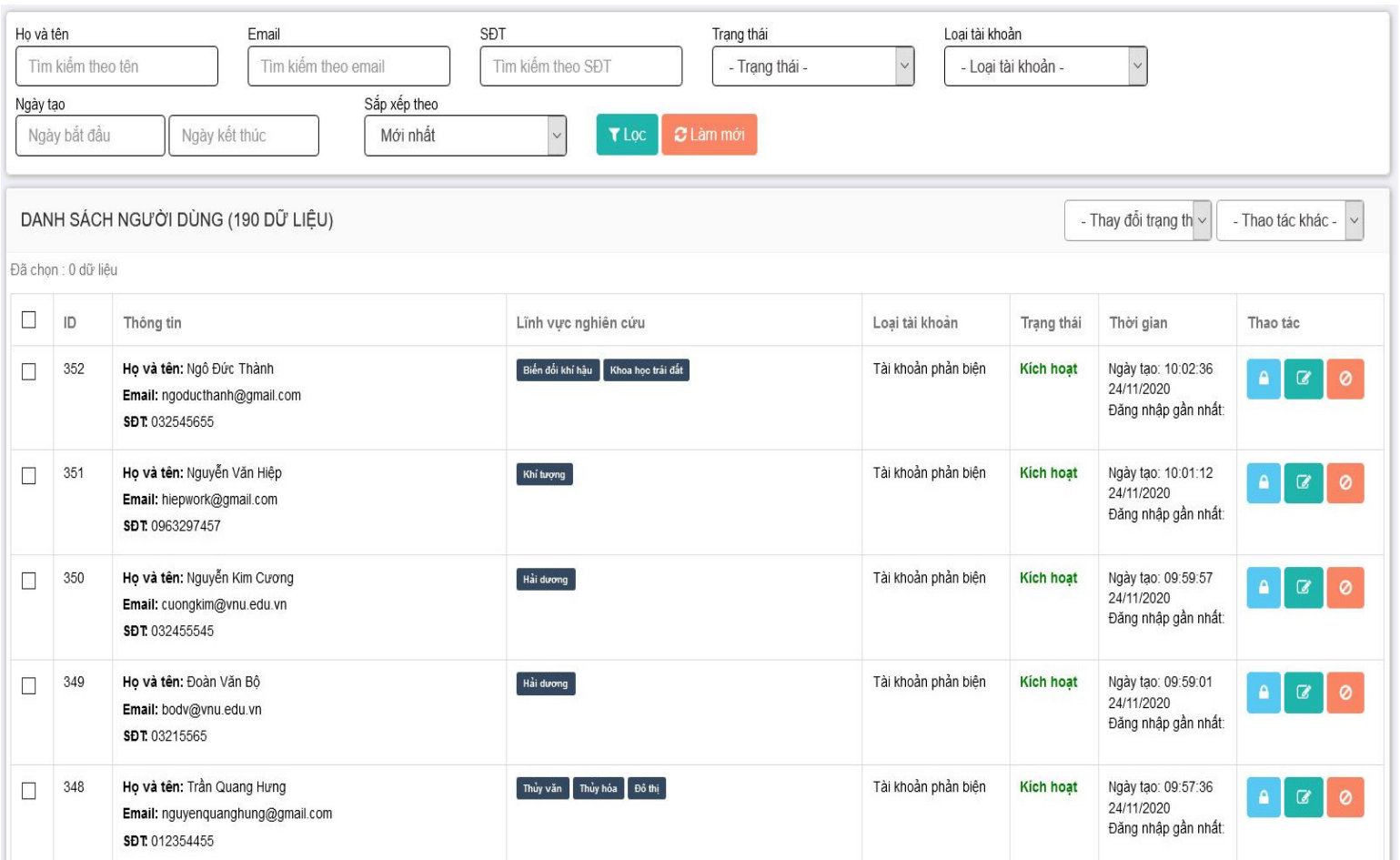

Hình 12. Giao diện danh sách người dùng.

Các tài khoản của tác giả và nhà phản biện đều được lưu trữ trong danh sách người dùng. Ban biên tập có thể quản lý, tìm kiếm người dùng theo họ tên, email, số điện thoại ... Đồng thời có thể tạo lại mật khẩu, chỉnh sửa và vô hiệu hóa tài khoản nếu nhận được yêu cầu từ phía người dùng (Hình 12). 


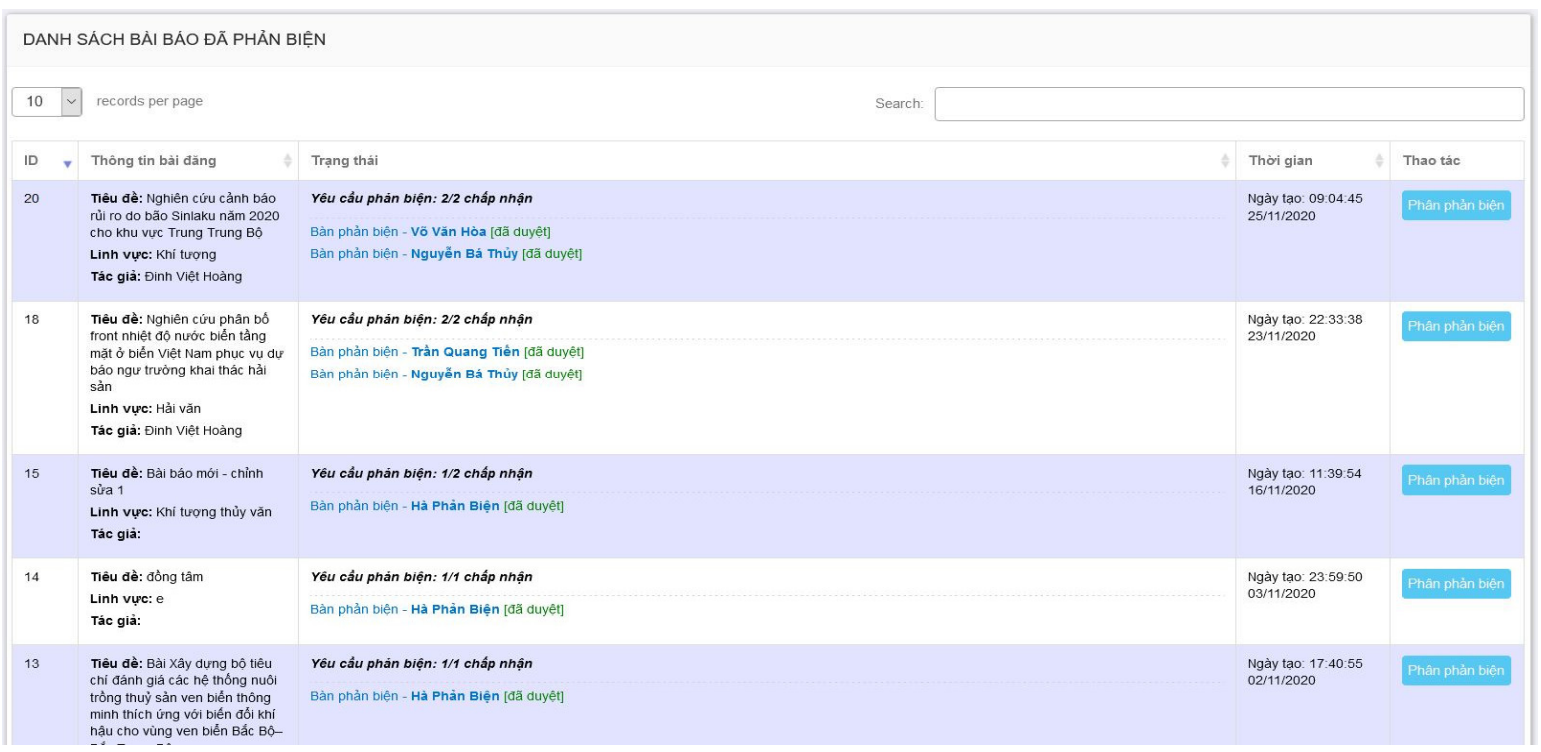

Hình 13. Giao diện danh sách bài báo đã phản biện.

Tất cả các bài báo trải qua quy trình xuất bản điện tử, sau khi kết thúc phản biện đều được lưu trữ tại danh sách các bài báo đã phản biện. Tại đây, Ban biên tập có thể dễ dàng theo dõi lại quá trình phản biện của từng bài báo, các yêu cầu chỉnh sửa của nhà phản biện, các bản chỉnh sửa và giải trình của tác giả (Hình 13 ). Kết quả của việc hoàn thiện quy trình xuất bản điện tử là minh chứng rõ ràng cho quá trình phản biện minh bạch trong công tác xuất bản điện tử của Tạp chí KTTV.

Các bài báo được xuất bản online đều xuất hiện trong mục bài báo trên trang chủ. Với giao diện thân thiện, rõ ràng, dễ theo dõi giúp các độc giả quan tâm dễ dàng tra cứu các thông tin về bài báo như là tiêu đề bài báo, thông tin tác giả, ngày xuất bản, chỉ số DOI, lượt tải, lượt xem và lượt trích dẫn (Hình 14). Độc giả có thể nhấn để xem các thông tin chi tiết hơn của bài báo như là nơi công tác của tác giả, tóm tắt, từ khóa, hướng dẫn trích dẫn và tài liệu tham khảo hoặc tải về bài báo đầy đủ.

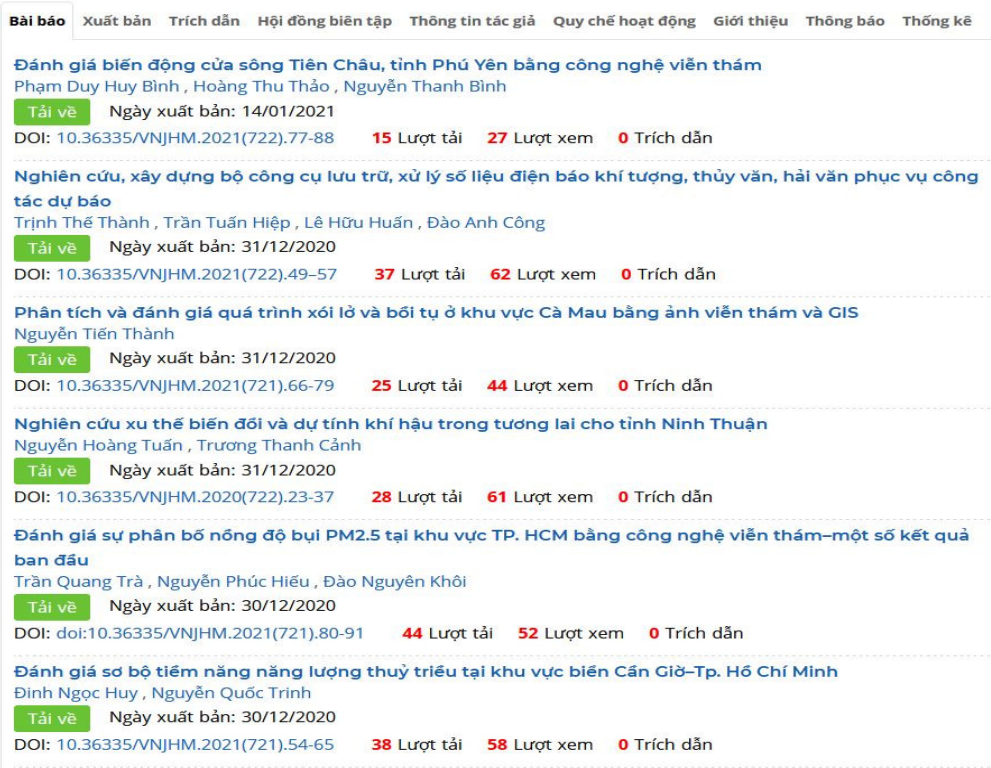

Hình 14. Giao diện bài báo trên trang chủ.

Hiện nay, Tạp chí KTTV đã đăng kí gia nhập thành công các hệ thống trích dẫn quốc tế: Google scholar [21], Scilit [22], CiteFactor [23], Publons [24] và hệ thống Tư liệu Khoa học Việt Nam CitationGate [25] của Đại học Quốc gia Hà Nội. Đây là những kênh thông tin rất 
quan trọng để độc giả, những nhà nghiên cứu, nhà quản lý trong và ngoài nước có thể tiếp cận và tìm đọc các bài báo khoa học của Tạp chí KTTV một các dễ dàng và thuận tiện hơn. Đây là bước khởi đầu rất quan trọng và từng bước khẳng định vị trí của Tạp chí KTTV trong quá trình hội nhập quốc tế.

Nhằm phục vụ tốt cho công tác thống kê, theo dõi số lượng bài báo xuất bản hàng năm, số lượng lượt tải, lượt xem và tổng số trích dẫn hàng năm, tỉ lệ rút nộp, tỉ lệ từ chối đăng, Tạp chí đã xây dựng một cơ sở dữ liệu thống kê dưới dạng biểu đồ cột và đường được thể hiện trên (Hình 15). Biểu đồ thống kê chỉ số trích dẫn theo năm thể hiện tổng số trích dẫn của các bài báo trong cùng một năm. Biểu đồ thống kê lượt xem tạp chí theo năm thể hiện tổng lượt xem của các bài báo trong cùng một năm. Biểu đồ thống kê lượt tải theo năm thể hiện tổng lượt tải của các bài báo trong cùng một năm. Biểu đồ thống kê số lượng bài báo theo năm thể hiện tổng số bài báo xuất bản trong một năm. Biểu đồ thống kê tỉ lệ rút nộp thể hiện tỉ lệ phần trăm số bài báo tác giả tự động rút nộp trong một năm. Biểu đồ thống kê tỉ lệ từ chối đăng thể hiện tỉ lệ phần trăm số bài báo bị từ chối đăng trong một năm. Dựa vào biểu đồ thống kê chỉ số trích dẫn theo năm và biểu đồ số lượng bài báo theo năm, tạp chí KTTV có thể dễ dàng tính được Impact Factor (chỉ số ảnh hưởng của tạp chí) theo từng năm.

\section{TẠP CHÍ KHÍ TƯợNG THỦY VĂN}
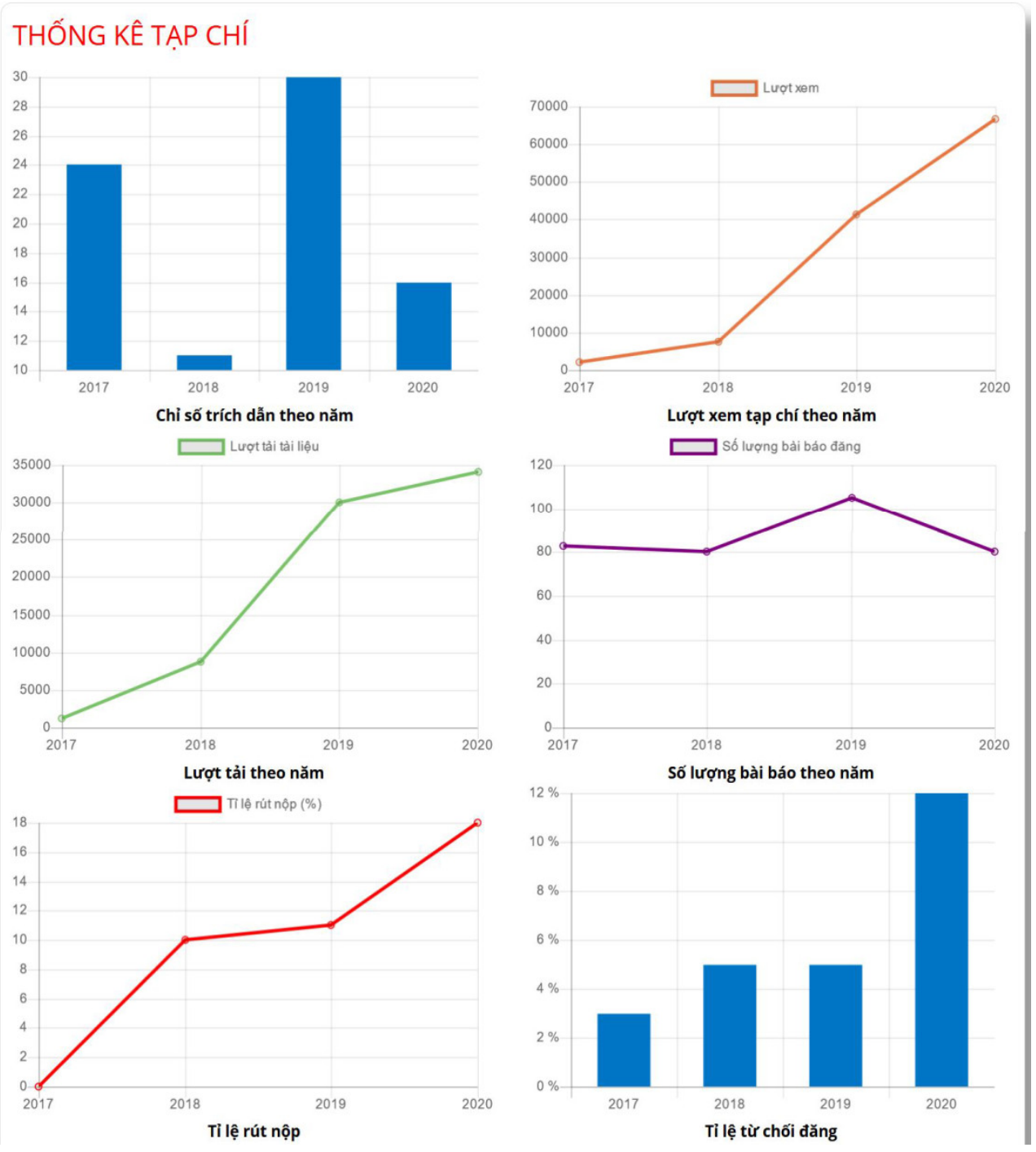

Hình 15. Giao diện số liệu thống kê Tạp chí. 


\section{Kết luận}

Công cụ quản lý và Quy trình xuất bản điện tử tại Tạp chí KTTV sau khi đi vào hoạt động, các Công cụ quản lý và Quy trình xuất bản điện tử đã thể hiện tính chuyên nghiệp, hiệu quả của mình so với công tác gửi bài, nhận bài và phản biện thông qua hòm thư điện tử trước đây. Công cụ quản lý dành cho tác giả, Ban biên tập và nhà phản biện với giao diện trực quan, dễ sử dụng thực hiện công tác xuất bản theo đúng quy trình khép kín và đảm bảo rõ ràng, minh bạch trong toàn bộ quy trình xuất bản. Toàn bộ các bài báo, tài khoản tác giả, độc giả, tài khoản nhà phản biện, quá trình phản biện đều được lưu trữ đầy đủ, tách biệt trong phần quản trị giúp Ban biên tập dễ quản lý và khai thác. Quá trình xuất bản online, các bài báo khoa học đã được phân tách thành từng bài riêng biệt và cập nhật trên trang chủ giúp cho các tác giả, độc giả dễ dàng theo dõi, tìm kiếm và sử dụng một cách hiệu quả hơn. Các số liệu về trích dẫn, lượt tải, lượt xem, tổng số bài báo, tỉ lệ rút nộp, tỉ lệ từ chối đăng cũng được thống kê chi tiết và đầy đủ theo từng năm dưới dạng biểu đồ để thuận tiện cho việc theo dõi quá trình xuất bản của Tạp chí KTTV.

Đóng góp của tác giả: Xây dựng ý tưởng nghiên cứu: Đ.Q.T., Đ.V.H.; Lựa chọn phương pháp nghiên cứu: Đ.V.H.; Viết bản thảo bài báo: Đ.V.H.; Chỉnh sửa bài báo: Đ.Q.T., Đ.V.H.

Lời cảm ơn: Nghiên cứu này là kết quả của đề tài nghiên cứu khoa học cấp cơ sở: "Nghiên cứu xây dựng công cụ quản lý, khai thác thông tin, dữ liệu phục vụ công tác truyền thông và xuất bản ấn phẩm điện tử”, mã số CS.2020.05.05.

Lời cam đoan: Tập thể tác giả cam đoan bài báo này là công trình nghiên cứu của tập thể tác giả, chưa được công bố ở đâu, không được sao chép từ những nghiên cứu trước đây; không có sự tranh chấp lợi ích trong nhóm tác giả.

\section{Tài liệu tham khảo}

1. Nhà xuất bản Springer: Truy cập https://www.springernature.com

2. Nhà xuất bản Elsevier: Truy cập https://www.elsevier.com

3. Nhà xuất bản Wiley-Blackwell: Truy cập https://onlinelibrary.wiley.com

4. Nhà xuất bản Taylor\&Francis: Truy cập https://www.tandfonline.com

5. Nhà xuất bản Sage: truy cập https://journals.sagepub.com

6. Nhà xuất bản Oxford University Press: Truy cập https://academic.oup.com

7. Nhà xuất bản Cambridge University Press: Truy cập https://www.cambridge.org

8. http://thuvien.uit.edu.vn/News/NewDetail/danh-sach-tap-chi-quoc-te-thuocdanh-muc-sci-scie-ssci-a-hci-isi

9. Tạp chí Nghiên cứu Kinh tế và Kinh doanh Châu Á: http://jabes.ueh.edu.vn/?lang=vi

10. Hệ thống Tạp chí của Đại học quốc gia Hà Nội: https://js.vnu.edu.vn/

11. Tạp chí điện tử Khoa học và công nghệ: http://tapchikhcn.udn.vn/home

12. Vietnam Journal of Earth Sciences: http://vjs.ac.vn/index.php/jse

13. Tạp chí Khí tượng Thủy văn: http://tapchikttv.vn/

14. Vietnam Journal of Hydrometeorology: http://vnjhm.vn/

15. Hội đồng Giáo sư nhà nước, Quyết định số 18/QĐ-HĐGSNN ngày 30 tháng 6 năm 2020 về việc Phê duyệt Danh mục tạp chí khoa học được tính điểm năm 2020 của Hội đồng Giáo sư liên ngành Khoa học Trái đất - Mỏ. http://tapchikttv.vn/public/upload/files/motraidat.pdf

16. Tỵ, N.Đ.; Phúc, Đ. Giáo Trình Cơ Sở Dữ Liệu. NXB Đại Học Quốc Gia, 2010.

17. Schwartz, B.; Zaitsev, P.; Tkacbenko, V.; Zawodny, J.D.; Lentz, A.; Balling, D.J. High Performance MySQL: Optimization, Backups, Replication, and More. Pub O'Reilly Media, 2008, pp. 710.

18. Schlossnagle, G. Advanced PHP Programming, Pub Sams Publishing, 2004. 
19. Duckett, J. HTML \& CSS: Design and Build Websites. Pub John Wiley \& Sons, Inc., Indianapolis, Indiana, 2011, pp. 514.

20. Elliott, E. Programming JavaScript Applications: Robust Web Architecture with Node, HTML5, and Modern JS Libraries. Pub O'Reilly Media, 2014, pp. 254.

21. Cơ sở dữ liệu Google scholar Tạp chí Khí tượng Thủy văn: https://scholar.google.com.vn/citations?view_op=list_works\&hl=vi\&authuser=4\&u ser $=$ JapFyGYAAAAJ\&gmla $=$ AJsNF7O31No53Rsf93bM_06hB9mMBF9W0pbBi9qVc7LoJmAvVJNqRo1vsWfzStGWqkkK4MpBhrefUUf2ylTc9iRWEWTf4MtLNg_uX9kSVOtYNoi7Vh0jv JdaR3OcI7frP3UpMCvARe

22. Cơ sở dữ liệu Scilit Tạp chí Khí tượng Thủy văn: https://www.scilit.net/article/468a9cf9f7aa948617f397f6dc0d06b2

23. Cơ sở dữ liệu CiteFactor: https://www.citefactor.org/journal/index/26081\#.YDsgJ2gzaiO

24. Cơ sở dữ liệu Publons: https://publons.com/researcher/3823695/vietnam-journalof-hydrometeorology/

25. Tư liệu Khoa học Việt Nam: https://vcgate.vnu.edu.vn/statistics/journals/tap-chikhi-tuong-thuy-van

\section{Research on a toolkit on information management and retrieval for electronic publication of Vietnam Journal of Hydrometeorology}

\section{Doan Quang Tri' ${ }^{1}$ Dinh Viet Hoang ${ }^{2 *}$}

1 Vietnam Journal of Hydrometeorology, Viet Nam Meteorological and Hydrological Administration; doanquangtrikttv@gmail.com; hoang.ceid@gmail.com

Abstract: Technological development and international integration have put forwards the requirements to improve the management and electronic publishing process of the Journal of Meteorology and Hydrology (KTTV). The electronic publishing process is modeled with PHP programming language, which store users' profile, article drafts, users' appraisal through MySQL database management system. Management tools with manuals and interface using HTML in combination with CSS and Javascript. The research results have successfully built an e-publishing process with supportive management tools for authors, the editorial board, reviewers, the editor-in-chief in e-publishing of Vietnam Journal of Hydrometeorology. Management tools and electronic publishing process show the suitability and efficiency in electronic publishing of the Journal.

Keywords: Management tools; Electronic publishing; Vietnam Journal of Hydrometeorology. 